\title{
Introduction: The philosophy of logical consequence and inference
}

\author{
Sten Lindström • Erik Palmgren • \\ Dag Westerståhl
}

Received: 11 September 2012 / Accepted: 11 September 2012 / Published online: 19 September 2012

(C) Springer Science+Business Media Dordrecht 2012

There is a traditional picture of logic that may be spelled out roughly as follows: Logic is concerned with the principles for correct reasoning and valid arguments; its principles are universal, necessary, a priori and formal; logically valid argument are necessarily truth-preserving and have a fundamental epistemic significance; and finally, logic is in some sense a normative discipline.

This traditional picture might appear appealing but it is at the same time deeply problematic and gives rise to many questions. The notions of universality, logical necessity, aprioricity and formality are notoriously difficult to analyse. In what sense, if any, is logic normative? Is there a principled way of distinguishing between logical and non-logical concepts? Is there a way of understanding logical consequence and logical inference that is compatible with the traditional picture?

While continuing to face these foundational questions, logic has developed into an advanced mathematical discipline — mathematical logic — where the informal notions of logical proof, validity and logical consequence are given rigorous mathematical explications. In mathematical logic, there are two major kinds of approaches to these notions: model-theoretic and proof-theoretic ones.

\footnotetext{
S. Lindström $(\varangle)$

Department of Historical, Philosophical and Religious Studies, Umea University, Umeå, Sweden e-mail: sten.lindstrom@philos.umu.se

E. Palmgren

Department of Mathematics, Stockholm University, SE-10691, Stockholm, Sweden

D. Westerståhl

Department of Philosophy, Stockholm University, Stockholm, Sweden

D. Westerståhl

Department of Philosophy, Linguistics, and Theory of Science, University of Gothenburg, Gothenburg, Sweden
} 
In model theory, logical consequence is explicated as truth-preservation in all (admissible) models of a given language. Usually, truth in a model is defined recursively à la Tarski. Within the model-theoretic approach, model-theoretic logical consequence is the fundamental notion and the notion of formal derivability (explicating the corresponding informal notion of logical deducibility) is secondary to the modeltheoretic notion, i.e. a system of formal logic is required to be sound, and if possible, also complete with respect to the corresponding model-theoretic semantics. A fundamental question in this connection: Is model-theoretic consequence a good explication of the (or one) informal, intuitive notion of logical consequence?

Within proof-theoretic semantics, the concept of a (canonical) proof, rather than truth-in-a-model, is fundamental. The notions of logical truth and consequence are defined in terms of this notion. This approach goes back to the Brouwer-HeytingKolmogorov interpretation of the logical connectives as well as to Gentzen's prooftheoretic analyses of logical inference in the 1930s. Proof-theoretic semantics can be viewed as a version of a more general programme, inferentialism. Inferentialist approaches to logic start out from the intuition that the meaning of a term, for example, a logical constant, is given by its inferential role. This is contrary to the classical truth-theoretic intuition underlying model-theoretic semantics according to which meaning is explained in terms of truth-conditions, and meaning in turn explains use and inferential role.

Model-theoretic as well as inferentialist and proof-theoretic approaches are faced with philosophical problems: To what extent do they correspond to our pre-theoretic and intuitive requirements on logical consequence? Which requirements should we expect an explication of logical consequence to meet? Is there only one satisfactory explication of the notion of logical consequence or are there several equally good ones? In particular, are the model-theoretic and proof-theoretic analyses really competitors or may they be seen as different approaches that complement each other?

These and related questions were discussed at a workshop held at the Swedish Collegium for Advanced Study (SCAS) in Uppsala, October 31-November 2, 2008. The workshop was organized by SCAS in cooperation with the Swedish National Committee for Logic, Methodology and Philosophy of Science and the Departments of Mathematics and Philosophy at Uppsala University. The papers presented at the workshop were:

- Hourya Benis Sinaceur: Tarski's notion of logical consequence and its interpretations

- Matti Eklund: On rivalry between logics

- Nissim Francez: Proof-theoretic semantics for a natural language fragment

- Christopher Gauker: Validity without reference

- Sten Lindström: Consequence without truth

- Per Martin-Löf: Formal consequence: the notion itself and Tarski's metamathematical reconstruction of it

- Sara Negri: Does the deduction theorem fail for modal logic?

- Peter Pagin: Assertion, inference, and consequence

- Dag Prawitz: Explications of the notions of inference and consequence 
- Stephen Read: The medieval theory of consequence

- Tor Sandqvist: Three notions of logical validity

- Gabriel Sandu: Semantic arguments and logical consequence

- Peter Schroeder-Heister: The categorical and the hypothetical: A critique of certain fundamental assumptions of standard semantics

- Sören Stenlund: von Wright on the future of logic and philosophy

- Göran Sundholm: Inference, consequence, conditional, implication

- Dag Westerståhl: Consequence and constancy

The present collection grew out of this workshop, but is not intended as a volume of proceedings. Our idea has instead been to collect some basic papers that will shed light on central topics that were discussed at the workshop.

A central theme in several of these papers concerns the distinction between logical consequence as a relation between sentences or propositions, and logical inference as an act, where a cognitive subject advances from asserting the premises to asserting the conclusion. For logicians working within the classical model-theoretic tradition, this distinction does not amount to much, since the notion of a valid inference is reduced to that of model-theoretic consequence: an inference is valid just in case its conclusion is a model-theoretic consequence of its premises. For other logicians, especially for those working within the intuitionistic tradition, the distinction between an epistemic notion of inference and a non-epistemic ("alethic") notion of logical consequence is crucial. In his contribution to this volume, Göran Sundholm clarifies the distinction between consequence and inference and discusses how it has been treated historically by, among others, the Scholastics, Bolzano, Frege, Gentzen and Martin-Löf. Dag Prawitz is concerned with explicating the notions of (epistemic) ground and deductively valid inference so that the following relationship holds as a conceptual truth: One gets possession of a ground for a conclusion by inferring it-by a valid inference-from premises for which one already has grounds. Peter Pagin gives an alternative characterization of valid inference in terms of information-preserving methods of inference. This notion is contrasted with (logical) consequence that is defined in terms of truth-preservation. Peter Schroeder-Heister criticizes certain fundamental assumptions that are shared by the model-theoretic and the intuitionistic traditions. In particular he rejects the transmission view of consequence, according to which consequence is defined in terms of the transmission of some basic categorical concept from the premises to the conclusion, be it truth, canonical provability, or some other concept. Instead he proposes an alternative view, according to which consequence is a primitive concept, which is not explained as the transmission of some categorical concept.

Dag Westerståhl's paper concerns the relationship between logical consequence and the notion of a logical constant. Standardly the definition of logical consequence for a language is taken to depend on a prior distinction between extra-logical symbols, whose interpretation is allowed to vary, and logical constants whose interpretation is fixed. In Westerståhl's paper the procedure is reversed: a method is given for extracting from an arbitrary consequence relation its associated set of logical constants.

Julien Boyer and Gabriel Sandu compare two kinds of verificationist definitions of truth for a statement, namely Dummett's definition in terms of the possibility of finding a constructive proof and Hintikka's definition in terms of the existence of a (recursive) 
winning strategy (for the Verifier) in the semantic game associated with the statement. The authors apply the distinction between the two kinds of verificationist semantics to a recent debate about how we can come to recognize the truth of Gödel sentences.

Tor Sandqvist's paper offers an interpretation of Gentzen-type multiple-conclusion sequents as a kind of meta-inference rules: a multiple conclusion inference rule represents a certain kind of inference from single-conclusion sequents.

Many authors have claimed that the deduction theorem does not hold for normal systems of modal logic with the rule of necessitation, while others claim the opposite. This situation is analysed in the paper by Raul Hakli and Sara Negri. They point out that when a Hilbert-style formalization of a system with the necessitation rule is generalized to a system that allows inferences from assumptions, the necessitation rule has to be modified accordingly, i.e., it has to be restricted in such a way that it does not allow the (unsound) inference from a formula that depends on assumptions to its necessitation. With this restriction of the necessitation rule, derivability from assumptions can be defined in the standard way and the deduction theorem will hold for the resulting system. The situation is analysed further by formulating the system $\mathrm{K}$ of basic modal logic as a sequent calculus.

Finally, there are two papers that take up issues in the philosophy of logic from a historical perspective. Stephen Read's contribution concerns various views of logical consequence that were developed during the Middle Ages. Matti Eklund discusses the interpretation of Rudolf Carnap's views of logic during the 1930s, in particular his logical pluralism and his famous Principle of Tolerance. In particular Eklund discusses critically certain interpretations of Carnap's views, due to Warren Goldfarb, Thomas Ricketts and Michael Friedman.

We thank the Swedish Collegium for Advanced Study (SCAS) in Uppsala for invaluable organizational support in connection with the workshop from which this volume has its origin. We are especially grateful to the Principal of SCAS, Professor Björn Wittrock, for supporting the project throughout and to the people at SCAS for all their efforts in connection with the workshop. We owe a special debt to Rysiek Sliwinski who was our co-organizer of the workshop. We gratefully acknowledge financial support from the Swedish Research Council and The Royal Swedish Academy of Sciences.

We should like to thank the reviewers of the individual papers for their efforts. Special thanks are due to Ingrid van Laarhoven and Jos Walbeek at Springer for excellent cooperation in connection with the production of this special issue. 